
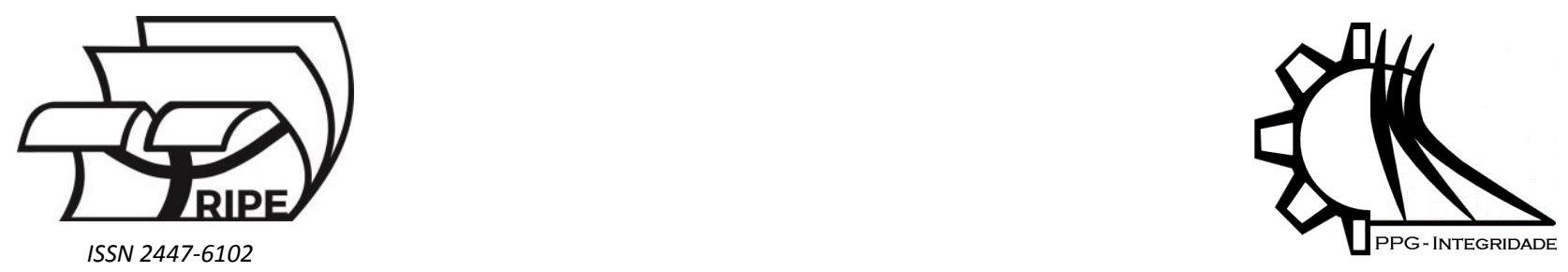

Article

\title{
Utilização da ferramenta ACP do ANSYS para análise de pilares de concreto armado confinados por PRFC
}

\author{
Silva Júnior, F.E.S. da ${ }^{1, *}$, Muniz, M.B.S. ${ }^{1}$ and Real, M. de V. ${ }^{1}$ \\ 1 Universidade Federal do Rio Grande \\ * Correspondence: jrsuri@outlook.com
}

Received: 14/12/2018; Accepted: 05/01/2019; Published: 29/01/2019

\begin{abstract}
Resumo: A utilização de polímeros reforçados com fibras (PRF) como método de reforço para estruturas de concreto tem ganhado destaque, sendo uma técnica que tende a ser amplamente utilizada por ser um método de reforço não destrutivo, eficiente e de fácil aplicação. No entanto, por ser uma técnica recente, muitas normas de diferentes países ainda não contemplam este tipo de reforço, tornando-se necessários mais estudos sobre o tema. Neste trabalho foram analisados seis pilares de concreto armado, dentre os quais, dois funcionaram como referência e quatro receberam reforço através da aplicação de polímero reforçado com fibra de carbono (PRFC) com variações quanto à orientação das fibras e à excentricidade da carga, com o objetivo de verificar a eficiência de cada tipo de confinamento para situações de compressão e flexo-compressão. Para a análise computacional, foi utilizado o software ANSYS com o auxílio do plugin ACP (ANSYS Composite PrepPost), comparando-se os resultados obtidos na simulação a resultados experimentais. Através da análise por elementos finitos, foi possível concluir que o reforço mostrou-se eficiente em todas as situações. Também foi possível observar que o comportamento das peças simuladas representou bem a realidade.
\end{abstract}

Palavras-chave: reforço estrutural, elementos finitos, ANSYS, fibra de carbono.

\section{Use of the ANSYS ACP tool for analysis of reinforced concrete columns wrapped by PRFC}

\begin{abstract}
The use of fiber-reinforced polymers (FRP) as a strengthening method for concrete structures has gained prominence, being a technique that tends to be widely used as a non-destructive, efficient and easy to apply strengthening method. However, because it is a recent technique, many standards from different countries do not yet contemplate this type of reinforcement, making further studies necessary on the subject. In this work six reinforced concrete columns were analyzed, two of which functioned as reference and four received strengthening through the application of carbon fiber reinforced polymer (CFRP) with variations in fiber orientation and load eccentricity, with the objective of verifying the efficiency of each type of confinement for situations of compression and flexural compression. For the computational analysis, the ANSYS software was used with the aid of the ACP plug-in (ANSYS Composite PrepPost), comparing the results obtained in the simulation to the experimental results. Through the finite element analysis, it was possible to conclude that the strengthening proved to be efficient in all situations. It was also possible to observe that the behavior of the simulated specimens represented reality well.
\end{abstract}

Keywords: structural strengthening, finite elements, ANSYS, carbon fiber.

\section{Introdução}

As exigências fundamentais para que uma edificação seja considerada adequada para uso são segurança, funcionalidade e durabilidade. Em estruturas de concreto armado, um reforço pode ser empregado para cumprir 
essas exigências quando ocorre um mau dimensionamento da estrutura, manifestações patológicas ou em decorrência de alterações no uso de uma edificação (devido ao alto custo envolvido em se demolir e refazer uma estrutura de concreto armado). Assim, o método de reforço por meio da aplicação de polímeros reforçados com fibras (PRF) tem se tornado cada vez mais utilizado.

Até recentemente, o método de reforço mais utilizado em estruturas de concreto era a aplicação de chapas de aço. Entretanto, isso mudou com a popularização dos PRF, que são materiais leves, resistentes à corrosão, possuem excelentes propriedades mecânicas e adaptam-se facilmente a qualquer superfície, além de não necessitarem de ferramentas diferenciadas para sua aplicação.

Apesar das propriedades favoráveis e da crescente utilização, alguns países ainda não possuem normas específicas para este tipo de reforço, tornando-se um tema relevante para estudo. Nos Estados Unidos, por exemplo, apenas em 2013 a norma ACl 562 (2013) introduziu recomendações sobre reforço e reparo em estruturas de concreto através de PRF.

Além das características citadas, uma propriedade pouco estudada e que também influencia na eficiência do confinamento é a orientação das fibras do PRF, que é objeto de estudo deste trabalho. Para isto, foi realizada, no software ANSYS, uma simulação computacional de seis pilares de concreto armado ensaiados por Muniz (2017). Os pilares foram separados em três grupos: o primeiro continha dois pilares sem reforço que funcionaram como referência para os demais; o segundo grupo apresentava dois pilares que receberam duas camadas de PRFC com as fibras na direção horizontal; e o terceiro grupo foi composto por dois pilares envolvidos por duas camadas de PRFC com fibras a 45 graus. Em cada grupo, um pilar recebeu carregamento concêntrico e o outro recebeu carga excêntrica. Para a definição da orientação das fibras foi utilizada a ferramenta ACP (ANSYS Composite PrepPost), disponibilizada juntamente com a plataforma Workbench do programa.

De acordo com Piscesa et al. (2018), uma das maiores dificuldades na modelagem numérica é lidar com a dilatação não-uniforme entre a parte interna do núcleo de concreto (envolta tanto pela armadura transversal como pelo PRF) e a parte externa (entre a armadura transversal e o PRF). Segundo Mousavi et al. (2010), o concreto apresenta maior resistência e capacidade de deformação axial quando é uniaxialmente carregado e não pode se dilatar lateralmente.

Sendo assim, o objetivo deste trabalho é representar computacionalmente o comportamento dos pilares ensaiados em laboratório e verificar a eficiência de cada tipo de confinamento para situações de compressão e flexo-compressão.

\section{Modelagem computacional}

Os seis pilares foram modelados conforme o experimento realizado por Muniz (2017) e foram analisados de forma não-linear. A modelagem computacional foi realizada no software ANSYS Workbench 17.1 (versão Student). Para realização do estudo, foram modelados os pilares com base quadrada com $10 \mathrm{~cm}$ de lado e $50 \mathrm{~cm}$ de altura.

Foram utilizadas quatro barras de aço para a armadura longitudinal, e foram inseridos três estribos em cada extremidade e três estribos intermediários, com detalhamento mostrado na Fig. 1. Todas as barras de aço tinham diâmetro igual a $8 \mathrm{~mm}$. Apesar de os pilares ensaiados por Muniz (2017) terem tido suas arestas arredondadas para receber o reforço por PRFC, este arredondamento não foi feito na modelagem computacional para fins de simplificação do modelo.

Foi utilizado o elemento tridimensional Solid65 para o concreto e definida resistência à compressão $\left(f_{c}\right)$ de 24,95 $\mathrm{MPa}$, e adotado o valor de resistência à tração $\left(f_{c t, m}\right)$ igual a 2,56 MPa. Foi adotado o valor de 0,20 para o coeficiente de Poisson e 27972 MPa para o módulo de elasticidade. O coeficiente de transferência de cisalhamento para fissuras abertas (open crack shear transfer coefficient) foi igual a 0,20 e o valor do coeficiente de transferência de cisalhamento para fissuras fechadas (closed crack shear transfer coefficient) foi igual a 1,00. Foi definido um gráfico multilinear de tensão-deformação do concreto sob compressão (Fig. 2) e seu comportamento foi considerado isotrópico. 

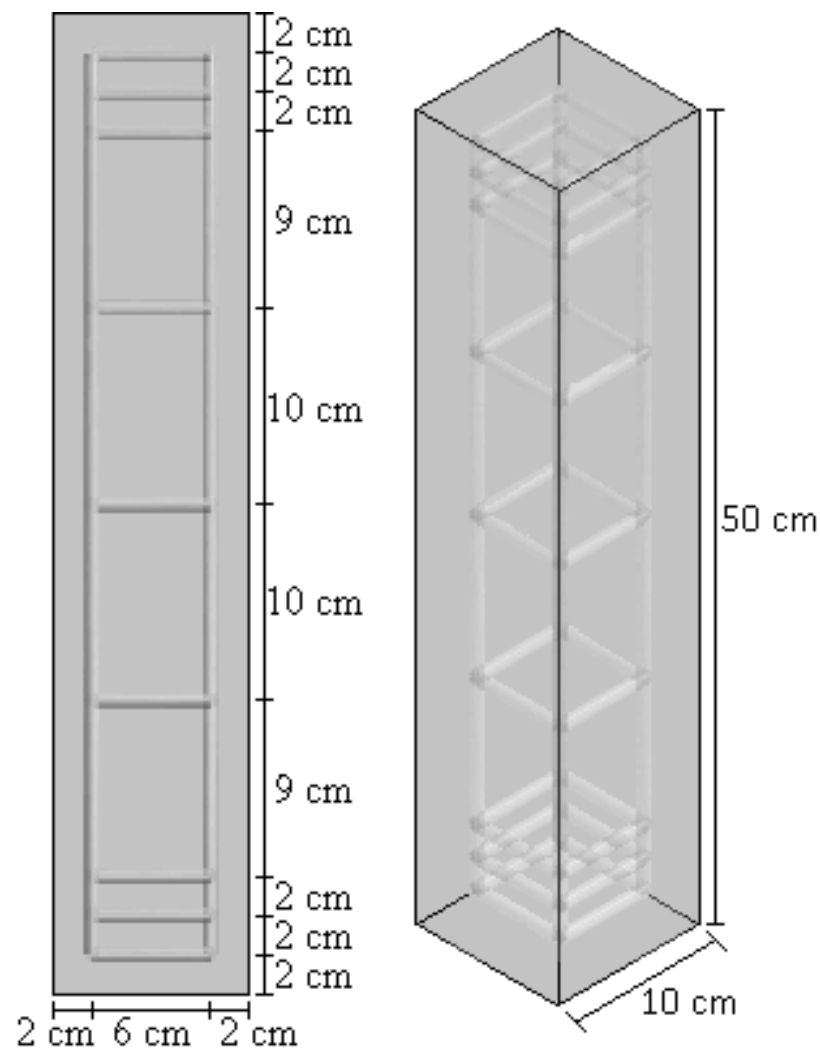

Figura 1. Detalhamento da modelagem das armaduras

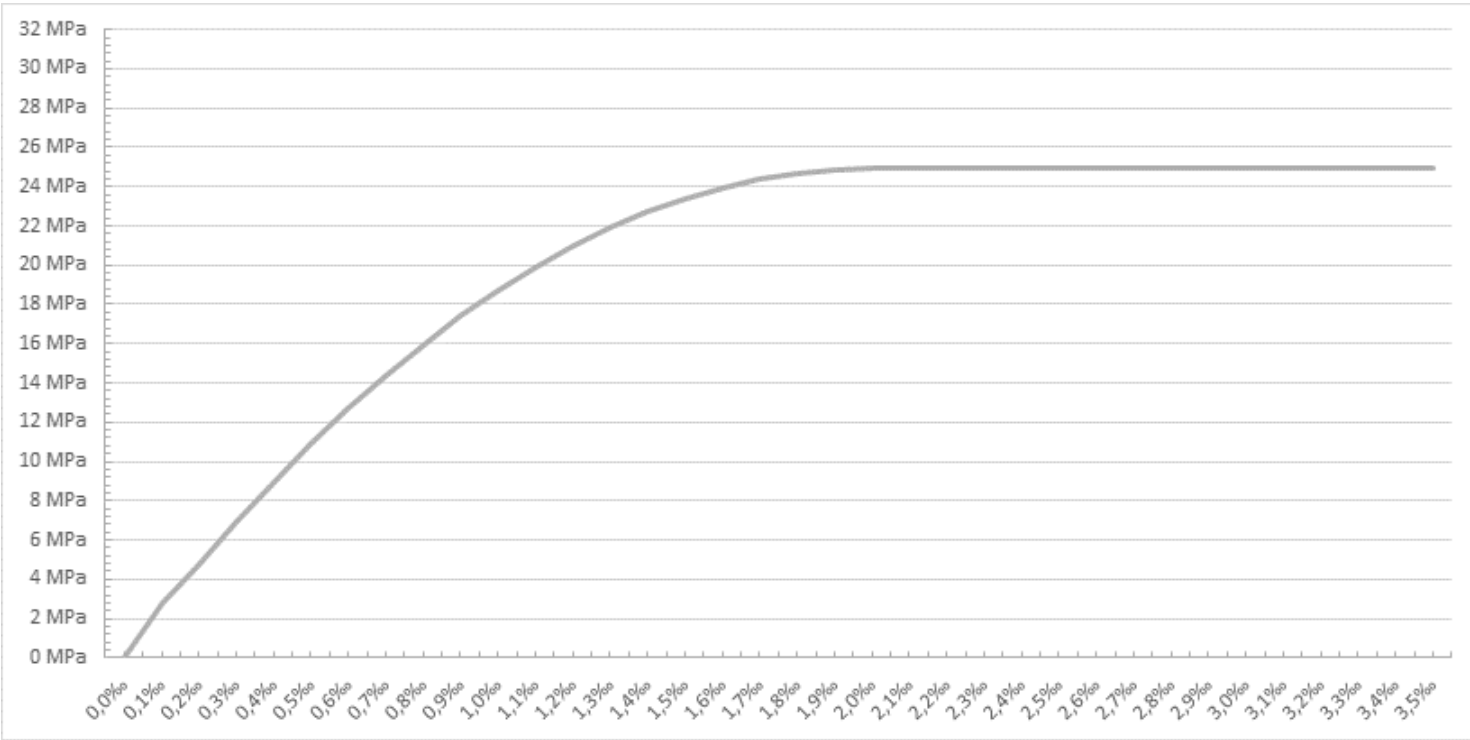

Figura 2. Gráfico tensão x deformação do concreto comprimido.

Segundo Ayala (2017), a rigidez da armadura, dependendo da idealização, pode ser incluída no modelo de elementos finitos de forma incorporada, distribuída ou discreta (Fig. 3). No caso da armadura incorporada, a mesma é considerada como uma linha de material mais rígido com aderência perfeita no interior dos elementos. Já na modelagem distribuída, a armadura é representada por um modelo de discretização em camadas, onde o concreto é uniformemente armado dentro de cada elemento finito. E na modelagem discreta é possível simular a falta de aderência entre concreto e armadura, e a armadura deve ser posicionada na região de encontro entre os elementos do concreto (arestas), de modo a se coincidir todos os nós dos elementos do concreto e do aço. Como o elemento 
utilizado para o concreto (Solid65) é compatível apenas com armadura distribuída e discreta, nesta análise a armadura foi modelada de forma discreta, ou seja, com necessidade de se coincidir os nós dos elementos.

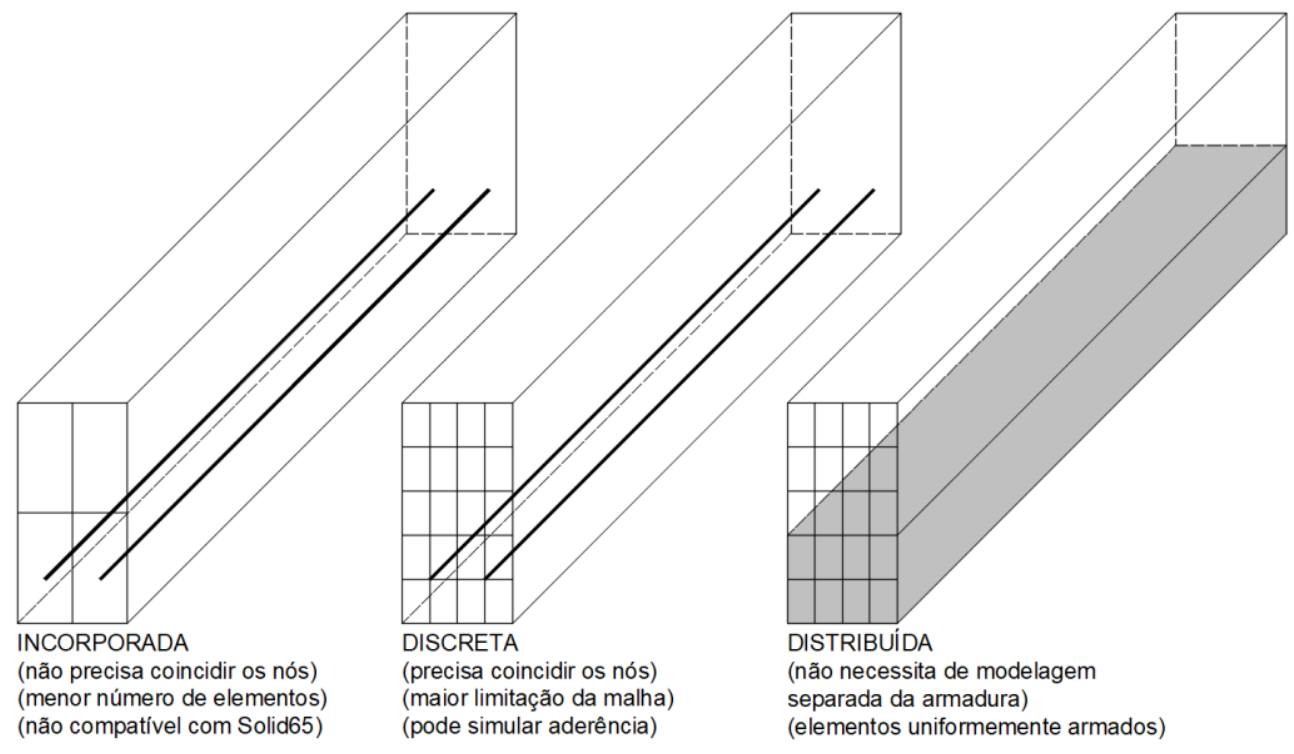

Figura 3. Modelos em elementos finitos para representação das armaduras.

Desta forma, a fim de se coincidir todos os nós, a malha de elementos finitos foi concebida com $2 \mathrm{~cm}$ nas direções X e Z e $1 \mathrm{~cm}$ na direção Y. Os apoios (reações) e pontos de aplicação de carga (ações) foram definidos na própria malha de elementos finitos, como mostrado nas Figs. 4(a)-(b), com distância de $2 \mathrm{~cm}$ entre os nós, de forma a idealizar um sólido com $2 \mathrm{~cm}$ de largura nesses pontos, assim como foi realizado no ensaio experimental.

Foi utilizado o elemento tridimensional Link180 para o aço das armaduras. Foi definido um gráfico bilinear para o escoamento do material e seu comportamento foi considerado isotrópico, e foi definida uma área de seção transversal igual a $50,24 \mathrm{~mm}^{2}$ para todas as barras. A tensão de escoamento do material foi igual a $500 \mathrm{MPa}$. $\mathrm{O}$ coeficiente de Poisson em XY foi igual a 0,3. O módulo de elasticidade foi igual a $210 \mathrm{GPa}$. O módulo tangente foi igual a $20 \mathrm{MPa}$ para evitar perda de estabilidade após o escoamento. A aderência entre concreto e armaduras foi considerada perfeita.

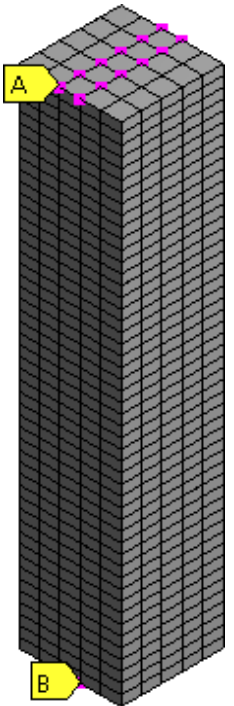

(a) Pilares sem excentricidade

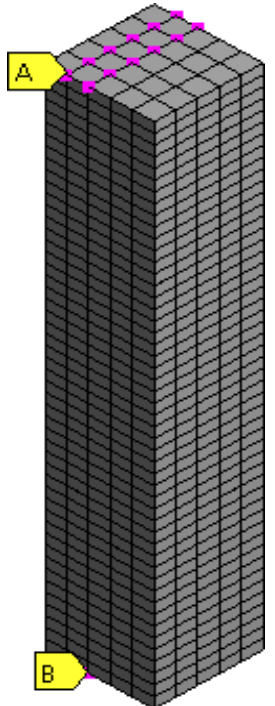

(b) Pilares com excentricidade $=2 \mathrm{~cm}$

Figura 4. Pontos de ação (A) e reação (B) nos pilares 
Foi definido o elemento Shell181 para o reforço. Por ser um elemento de casca e utilizar um material de comportamento ortotrópico, foi necessário definir a orientação das fibras, o que foi feito com o auxílio da ferramenta ACP (Fig. 5). Além disso, a malha utilizada para este elemento foi menor que a malha utilizada no concreto e na armadura, apresentando dimensões de $1 \mathrm{~cm}$ nas duas direções superficiais.
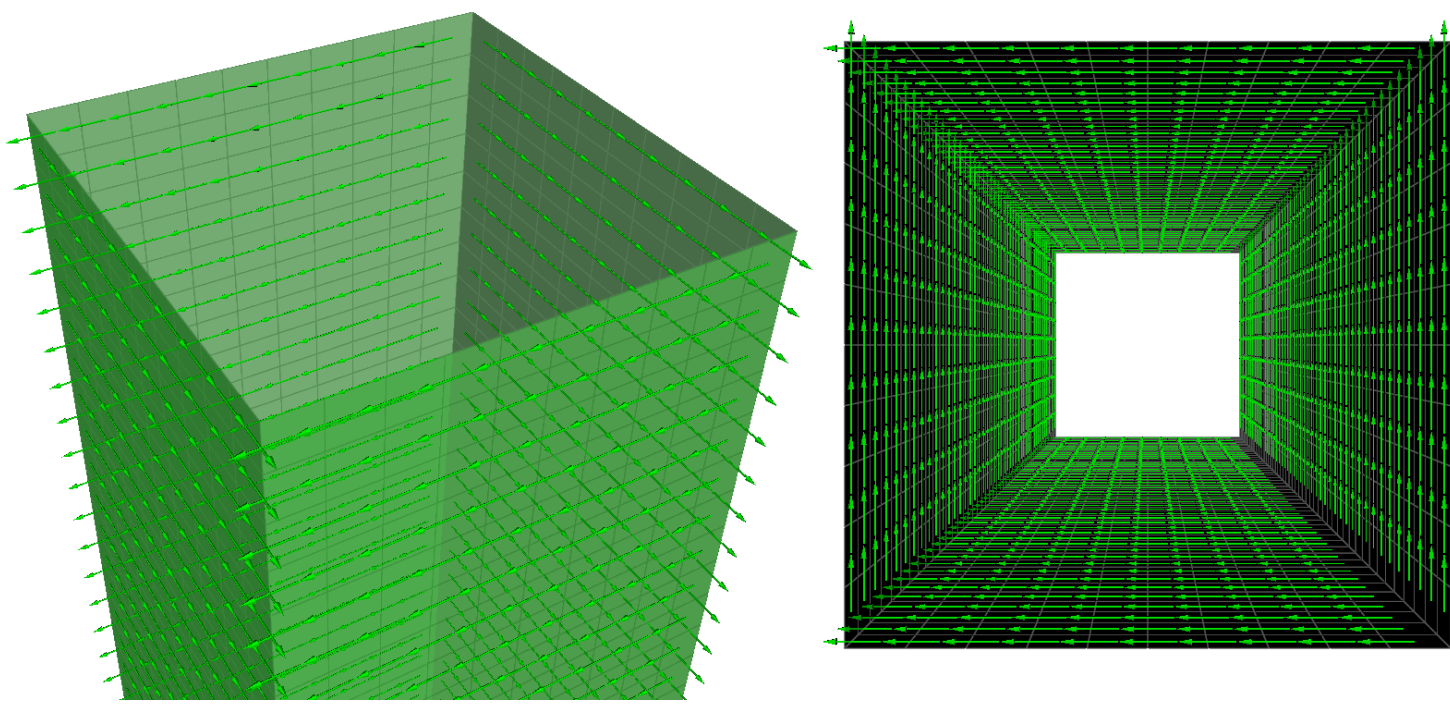

Figura 5. Definição da orientação das fibras de carbono no ACP: pilares com fibras na direção horizontal.

Na Tab. 1 são apresentadas as propriedades do material compósito definidas para a análise, as quais foram informadas pelo fabricante.

Tabela 1. Propriedades do PRFC

\begin{tabular}{cc}
\hline Fibras de carbono (direção X do compósito) \\
\hline Espessura da manta & $0,166 \mathrm{~mm}$ \\
Resistência à tração & $4900 \mathrm{MPa}$ \\
Módulo de elasticidade & $230 \mathrm{GPa}$ \\
Alongamento na ruptura & $2,1 \%$ \\
\hline
\end{tabular}

Na Fig. 6 é apresentado o esquema da orientação das fibras de cada pilar e o tipo de carregamento (concêntrico ou excêntrico). No caso dos pilares com fibras a 45 graus, no ensaio experimental foi aplicada uma camada em cada direção, formando uma espécie de trama. Entretanto, na simulação foi modelada apenas uma camada com o dobro de espessura e fibras em duas direções, a fim de se reduzir o enorme esforço computacional que seria empregado caso fossem modeladas duas camadas com fibras em direções diferentes. 

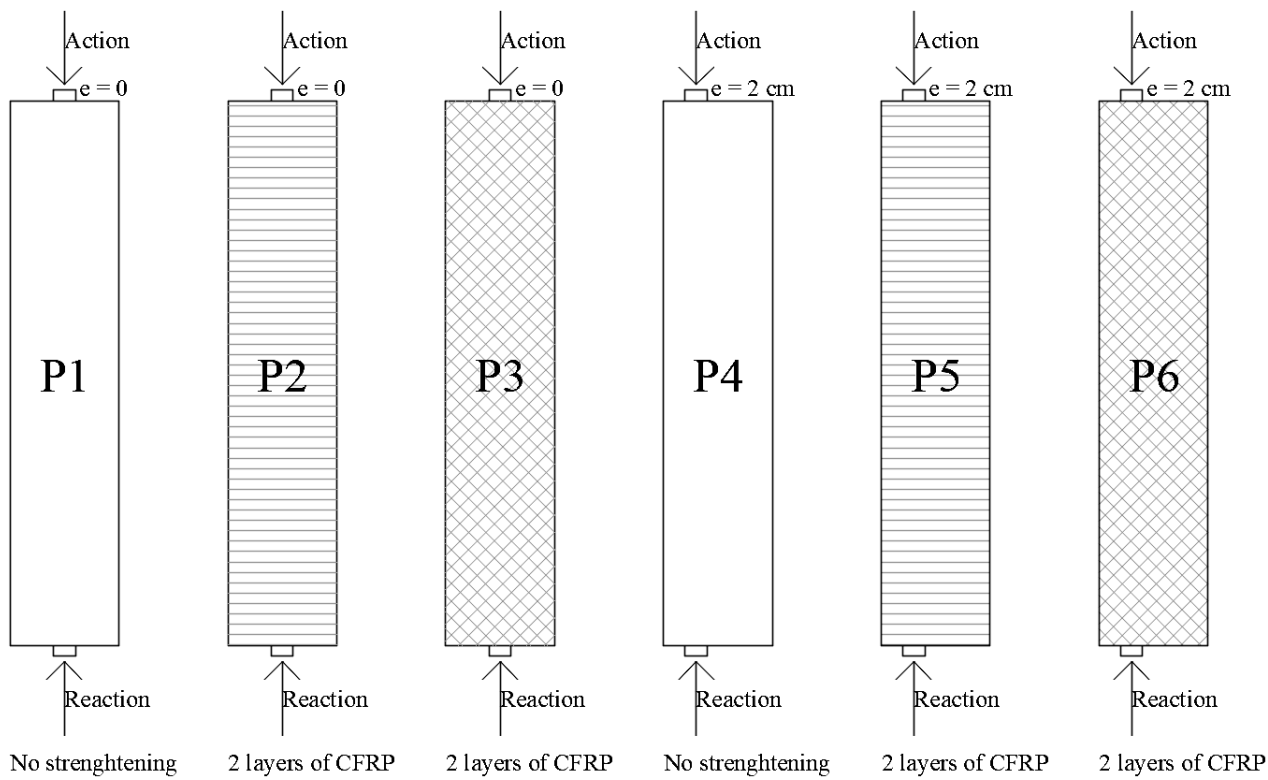

Figura 6. Orientação das fibras e tipo de carregamento em cada pilar

A análise foi realizada em um load step dividido em 100 substeps, e foi utilizado o método de Newton-Raphson completo para convergência baseada em força e deslocamento. Os apoios foram definidos como deslocamentos verticais nulos nos nós das reações, e foi aplicado deslocamento na direção -Y nos pontos de ação até o programa interromper a simulação retornando um erro de magnitude, indicando que o carregamento excedeu a capacidade resistente da peça.

\section{Resultados}

Na Tab. 2 são apresentados os resultados computacionais e os experimentais para fins de comparação. Nota-se que o PRFC conseguiu elevar a resistência de todas as peças em relação aos pilares de referência. Assim como no experimento, o confinamento do concreto através de fibras inclinadas foi menos eficiente que o confinamento por fibras horizontais, no caso de carga concêntrica.

Tabela 2. Resultados

\begin{tabular}{cccc}
\hline Pilar & Tipo & Carga & Carga \\
& Referência - carga centrada & (experimental) & (computacional) \\
\hline P1 & Fibras horizontais - carga centrada & 50,00 & 55,28 \\
P2 & Fibras a 45 - carga centrada & 89,00 & 87,99 \\
P3 & Referência - carga excêntrica (2 cm) & 67,00 & 74,65 \\
P5 & Fibras horizontais - carga excêntrica (2 cm) & 47,00 & 45,65 \\
P6 & Fibras a 45 ${ }^{\circ}$ - carga excêntrica $(2 \mathrm{~cm})$ & 63,00 & 69,73 \\
\hline
\end{tabular}

Através das Figs. 7 e 8 é possível notar que o método de aplicação do carregamento favoreceu a concentração de tensões próximo às regiões dos apoios devido ao modo que foi aplicada a ação, o que contribuiu para a redução da capacidade resistente das peças. 


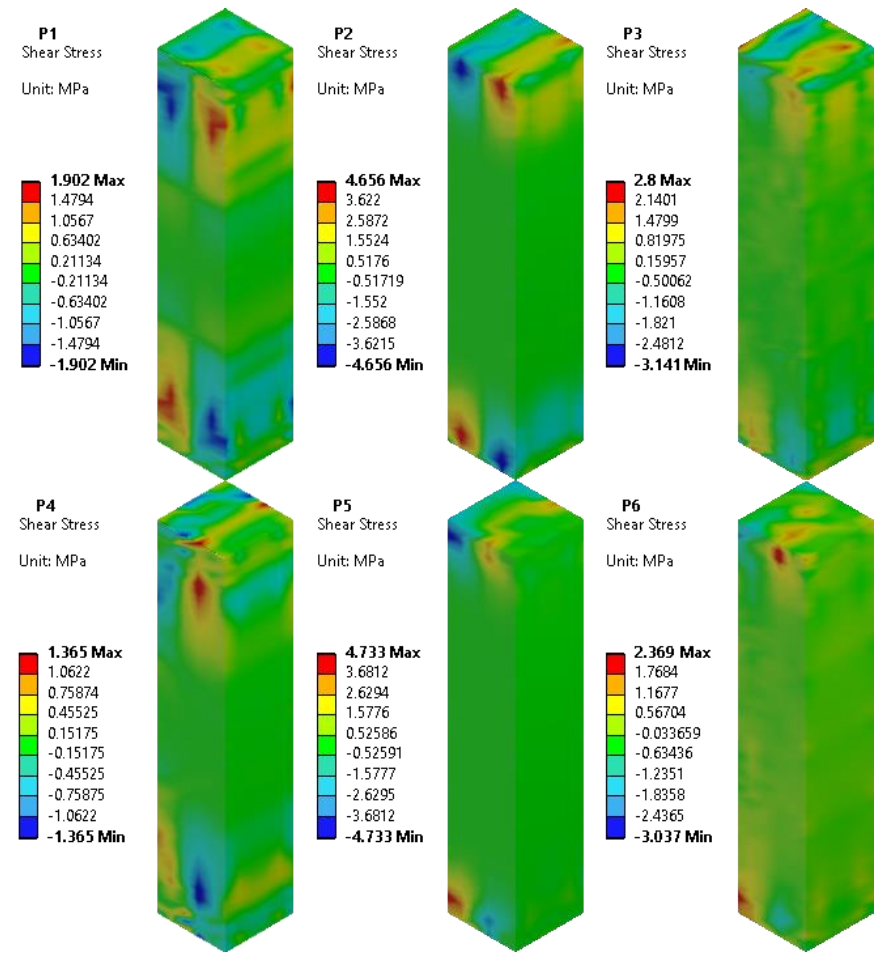

Figura 7. Tensões de cisalhamento.

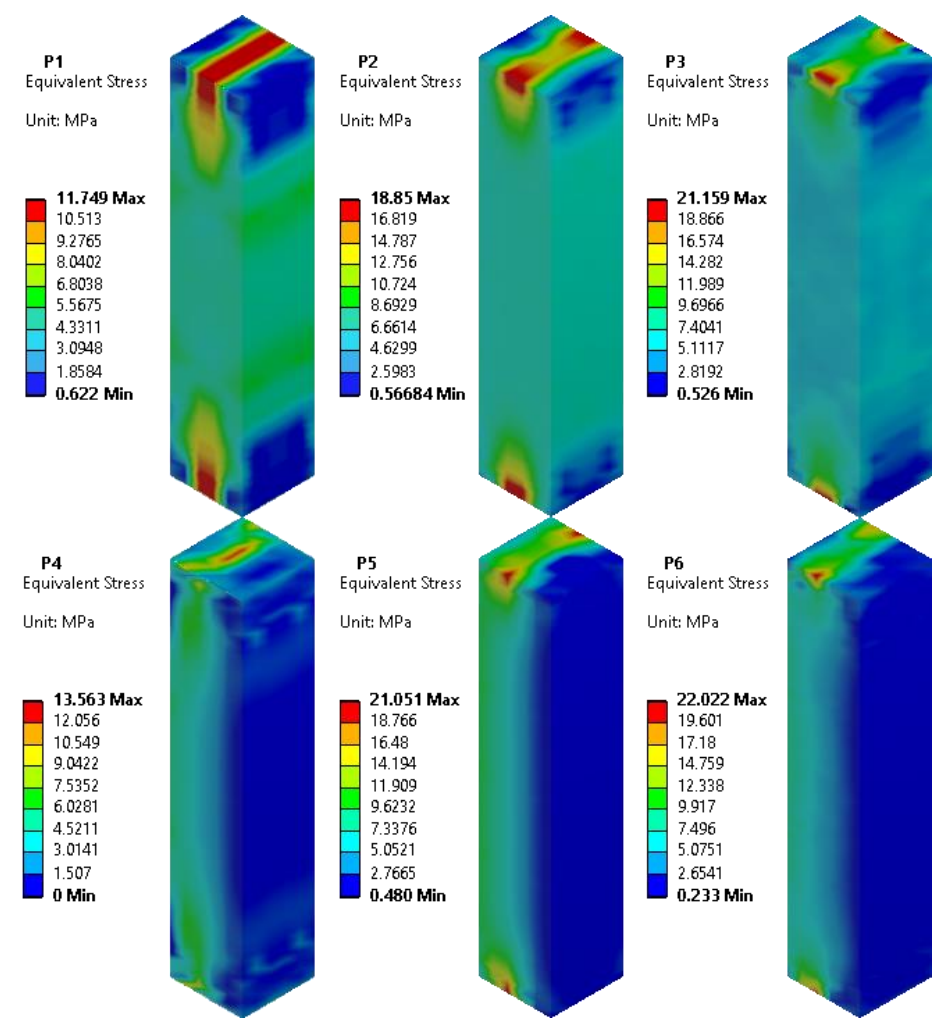

Figura 8. Caminhos de tensões (tensão equivalente).

Na Fig. 8 são apresentados os caminhos de tensões nas peças. Nos pilares com excentricidade, é possível observar um padrão de caminhos de tensões semelhante ao de uma viga submetida à flexão, devido à 
flexo-compressão atuando nestas peças. Estes caminhos de tensões coincidem com as regiões onde as fibras de carbono sofreram maior deformação específica (Fig. 9). E analisando a Fig. 9, percebe-se que, entre os pilares com carga centrada, houve maior deformação específica no PRFC do pilar P2 (fibras horizontais). Já entre os pilares com carga excêntrica, houve maior deformação no reforço do pilar P6 (fibras diagonais). Isto pode indicar que o reforço foi mais solicitado nestes espécimes antes de ocorrer a ruptura.

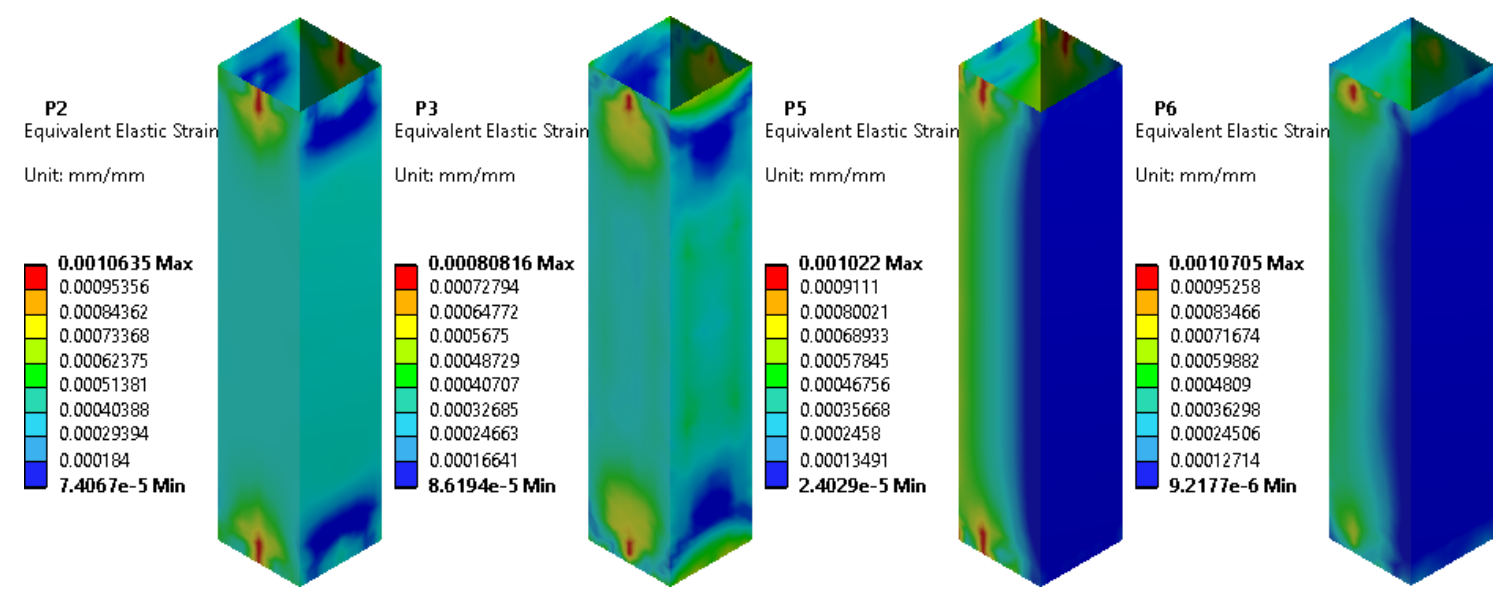

Figura 9: Deformação específica nos reforços

Os resultados obtidos no pilar P2, tanto experimentais quanto computacionais, divergem da afirmação de Chaallal e Shahawy (2000), que diz que quanto maior a angulação escolhida para orientação das fibras no confinamento do elemento estrutural, menor a resistência axial e pressão de confinamento da seção. Ao contrário disto, o método de reforço do pilar P2 (fibras a 90 em relação ao eixo do pilar) foi o mais eficaz, conferindo a este elemento a maior carga de ruptura entre os seis espécimes. Entretanto, quando se trata dos resultados computacionais dos pilares com carga excêntrica, nota-se que o P6 obteve menor resistência que o pilar P5, ao contrário do que ocorreu no laboratório. Uma hipótese para explicar esta variação é a de que ela possa ter ocorrido devido às simplificações de modelo realizadas na simulação e à concentração de tensões devido ao método de aplicação de carga, o que não invalida a simulação, pois ainda assim os resultados se mostraram próximos e confiáveis.

Assim como no ensaio experimental, os seguintes resultados foram constatados:

a) A orientação das fibras influenciou nos resultados;

b) Os pilares P1 e P4 foram os que obtiveram menores resistências, ou seja, a adição de fibras de carbono foi capaz de conceder maior capacidade resistente a todos os pilares reforçados em relação aos pilares de referência;

c) O pilar P2 foi o mais resistente, e seu método de confinamento foi mais eficiente que o do pilar P3;

d) Portanto, apesar de alguns autores como Lin e Teng (2017), Ferrotto et al. (2018) e Piscesa et al. (2018) afirmarem que o comportamento do concreto armado confinado não é bem representado por elementos finitos através de modelos atuais, os resultados da análise se mostraram satisfatórios, representando bem a realidade.

\section{Conclusões}

Através dos resultados obtidos na análise computacional e de sua comparação aos resultados obtidos em laboratório, chegou-se às seguintes conclusões em relação à simulação:

a) Os pilares de referência (sem reforço) obtiveram as menores cargas de ruptura. O confinamento por PRFC foi eficiente em todas as situações, mostrando-se mais eficiente nos pilares com as fibras na direção horizontal, o que diverge de estudos anteriores na área, como o de Chaallal e Shahawy (2000), que indicavam que fibras inclinadas deveriam conferir maior resistência às peças;

b) Os resultados conseguiram representar bem a realidade, indicando que a análise foi satisfatória, com uma maior diferença apenas no pilar P6;

c) Uma das conclusões no trabalho de Muniz (2017) sugere que a utilização de fibras inclinadas possa ajudar no combate a esforços de tração causados pela flexo-compressão com grande excentricidade. Devido à diferença entre a carga última do pilar P6 na simulação e no experimento, sugere-se que sejam 
realizados mais estudos, tanto experimentais quanto computacionais, para melhor investigar a eficiência do reforço com fibras inclinadas em situações de flexo-compressão.

\section{Referências}

1. American Concrete Institute $[\mathrm{ACl}]$. Code Requirements for Evaluation, Repair, and Rehabilitation of Concrete Buildings (ACl 562M-13) (2013).

2. Ayala, I. C. A. (2017). Customização do software ANSYS para análise de lajes de concreto protendido pelo método dos elementos finitos. Dissertação de Mestrado, Universidade Federal do Rio Grande do Sul, Porto Alegre, Rio Grande do Sul, Brasil.

3. Chaallal, O., \& Shahawy, M. (2000). Perfomance of fiber-reinforced polymer-wrapped reinforced concrete column under combined axial-flexural loading. ACl Structural Journal, 97(4), 659-668. https://www.doi.org/10.14359/7433

4. Ferrotto, M. F., Fischer, O., \& Cavaleri, L. (2018). A strategy for the finite element modeling of FRP-confined concrete columns subjected to preload. Engineering Structures, 173, $1054-1067$. https://doi.org/10.1016/i.engstruct.2018.07.047

5. Lin, G., \& Teng, J. G. (2017). Three-dimensional finite-element analysis of FRP-confined circular concrete columns under eccentric loading. Journal of Composites for Construction, 21(4). https://doi.org/10.1061/(ASCE)CC.1943-5614.0000772

6. Mousavi, S. M., Alavi, A. H., Esmaeili and M. A., \& Gandomi, M. (2010). A data mining approach to compressive strength of CFRP-confined concrete cylinders. Structural Engineering and Mechanics, 36(6), 759-783.

7. Muniz, M. B. S. (2017). Estudo experimental e teórico de pilares curtos de concreto armado reforçados por confinamento de CFRP. Monografia, Universidade Estadual Vale do Acaraú, Sobral, Ceará, Brasil.

8. Piscesa, B., Attard, M. M., \& Samani, A. K. (2018). 3D finite element modeling of circular reinforced concrete columns confined with FRP using a plasticity based formulation. Composite Structures, 194(15), 851-875. https://doi.org/10.1016/j.compstruct.2018.04.039 